\title{
Machine learning and systems genomics approaches for multi-omics data
}

Eugene Lin ${ }^{1,2,3}$ and Hsien-Yuan Lane ${ }^{1,4^{*}}$

\begin{abstract}
In light of recent advances in biomedical computing, big data science, and precision medicine, there is a mammoth demand for establishing algorithms in machine learning and systems genomics (MLSG), together with multi-omics data, to weigh probable phenotype-genotype relationships. Software frameworks in MLSG are extensively employed to analyze hundreds of thousands of multi-omics data by high-throughput technologies. In this study, we reviewed the MLSG software frameworks and future directions with respect to multi-omics data analysis and integration. Our review was targeted at researching recent approaches and technical solutions for the MLSG software frameworks using multi-omics platforms.
\end{abstract}

Keywords: Genomics, Pharmacogenomics, Single nucleotide polymorphisms, Machine learning, Multi-omics, Systems genomics

\section{Background}

Over the past few years, researchers and scientists have made remarkable progress in the interdisciplinary fields of precision medicine, data mining and predictive algorithms, bioinformatics, and computational medicine [1]. Machine learning and systems genomics (MLSG) approaches integrate multiple data types from multi-omics data by using data mining and predictive algorithms, pointing out that the MLSG approaches can support a more meaningful interpretation of phenotype-genotype relationships than an analysis using only a single data type. Therefore, there is an acute need for development of the MLSG software frameworks that can generate prediction of a given quantitative or categorical phenotype using next-generation multi-omic data [2].

Precision medicine, an emerging field of medicine, is becoming the cornerstone of medical practices with prospects of the customization of healthcare, which means medical decisions, practices, and treatments are tailored to individual patients [3]. The use of genomic biomarkers, such as multi-omics data, has played a major role in precision medicine in oncology and other

\footnotetext{
* Correspondence: hylane@gmail.com

${ }^{1}$ Graduate Institute of Biomedical Sciences, China Medical University, Taichung, Taiwan

${ }^{4}$ Department of Psychiatry, China Medical University Hospital, Taichung, Taiwan

Full list of author information is available at the end of the article
}

chronic diseases such as asthma [4], mental disorders [5, 6], and diabetes [7-9]. More specifically, patients are divided into groups by genetic variability and other biomarkers so that medications may be tailored to individual patients with similar or related genetic characteristics $[10,11]$. For example, accumulating evidence reveals that selected single nucleotide polymorphisms (SNPs) could be used as genetic markers to influence clinical treatment response and adverse drug reactions for antidepressants in patients with major depressive disorder [12-14]. With the advent of technology in multi-omics approaches such as genomics, proteomics, metabolomics, and epigenomics, we are able to employ materials or devices that can interact with biological systems at the molecular level and then target different molecules with high precision.

In big data science, machine learning methods are computer algorithms that can automatically learn to recognize complex patterns based on empirical data $[15,16]$. The goal of an machine learning method is to enable an algorithm to learn from data of the past or present and use that knowledge to make predictions or decisions for unknown future events $[17,18]$. In the general terms, the workflow for an machine learning method consists of three phases including build the model from example inputs, evaluate and tune the model, and then put the model into production in prediction-making. Some of the bestknown algorithms in machine learning methods include 
naive Bayes [19], C4.5 decision tree [20], artificial neural networks (ANNs) [21-23], support vector machine (SVM) [24], k-Means [25], k-nearest neighbors ( $\mathrm{kNN})$ [26], and regression $[27,28]$. There were some key emerging diagnostics studies for various diseases and treatments of significance for public health with consideration of machine learning methods, including applications in mental health [29-33], cancer [34-38], and pharmacogenetics [39-41].

In this review, we surveyed the MLSG software frameworks that could enable definite assessment of the phenotype-genotype interplay status by using multi-omics platforms. The MLSG software frameworks encompass the model-based integration (MBI), concatenation-based integration (CBI), and transformation-based integration (TBI) approaches (Table 1). Furthermore, we investigated some potential data reduction and feature selection approaches that can be leveraged together with the MLSG software frameworks. Finally, we summarized the future perspectives with respect to the MLSG approaches.

\section{Model-based integration approach}

First, we explored the MBI approach, which generates multiple models using different data types as training sets, and then generates a final model from the multiple models created during the training phase (Fig. 1). One advantage of the MBI approach is that this approach can merge predictive models from different data types and each data type can be assembled from a different set of patients with same phenotype [42].

In order to identify interactions between different levels of genomic data associated with certain disease or phenotype (for example, survival in ovarian cancer), the MBI approach can integrate multi-omics data, including, but not limited to, miRNA, methylation, gene expression, and copy number variation data. The MBI approach can then conduct the final multi-dimensional model from a particular machine learning algorithm (for example, Bayesian networks) with variables from the best models of each individual genomic dataset. Next, the MBI approach can compare the predictive power of the integration model with the one of the individual model from single level of genomic data to see whether the integration model can show the improvement. Finally, the MBI approach can obtain the best multi-dimensional model of all variables from multi-omics dimension as well as a balanced accuracy for the final model.

In the literature, the MBI approach encompasses the following computational frameworks for constructing a model: a majority voting approach [43], an ensemble classifier approach [44], and probabilistic causal networks [45]. In addition, we can employ the Analysis Tool for Heritable and Environmental Network Associations methodology, which is a suite of analysis tools for integrating multi-omics data [46].

\section{Probabilistic causal network framework}

In order to integrate highly dissimilar types of data, we can leverage Bayesian networks that are one type of probabilistic causal networks [47]. Bayesian networks are directed acyclic graphs where the edges of the graph are represented by conditional probabilities, which define the distribution of states of each node given the state of its parents [47]. In Bayesian networks, each node characterizes a quantitative trait that can be a genomic factor (such as variation in DNA, gene expression, methylation, metabolite, and protein). These conditional probabilities represent not only relationships between genomic factors, but also the stochastic nature of these relationships. By assuming the observed data as a function of our prior belief, the Bayes formula is used to determine the likelihood of a Bayesian network model. Because the number of potential network structures grows super-exponentially with the number of nodes, it is infeasible to find the best model by an exhaustive search of all possible structures. Therefore, we can utilize Monte Carlo Markov Chain simulation [48] to pinpoint probably a huge amount of different plausible Bayesian networks, which are then integrated to accomplish a consensus network model. In the beginning, there

Table 1 Summary, strength, and limitation of each method of machine learning and systems genomics (MLSG) software frameworks

\begin{tabular}{|c|c|c|c|}
\hline Software framework & Summary & Strength & Limitation \\
\hline $\begin{array}{l}\text { Model-based } \\
\text { integration (MBI) }\end{array}$ & $\begin{array}{l}\text { Multiple predictive models are generated } \\
\text { by using various multi-omics data types; } \\
\text { then a final predictive model is generated } \\
\text { by using the multiple models. }\end{array}$ & $\begin{array}{l}\text { Predictive models can be consolidated from } \\
\text { various multi-omics data types, and each data } \\
\text { type can be gathered from a various set of } \\
\text { patients with same phenotype. }\end{array}$ & $\begin{array}{l}\text { It may be challenging to avoid } \\
\text { overfitting. }\end{array}$ \\
\hline $\begin{array}{l}\text { Concatenation-based } \\
\text { integration }(C B I)\end{array}$ & $\begin{array}{l}\text { Multiple data matrices of different } \\
\text { multi-omics data types are incorporated } \\
\text { into a large input matrix; then a predictive } \\
\text { model is generated by using the large } \\
\text { input matrix. }\end{array}$ & $\begin{array}{l}\text { It is fairly easy to leverage various machine } \\
\text { learning methods for analyzing continuous } \\
\text { or categorical data once a large input matrix } \\
\text { is formed. }\end{array}$ & $\begin{array}{l}\text { It may be challenging to } \\
\text { combine a large input matrix. }\end{array}$ \\
\hline $\begin{array}{l}\text { Transformation-based } \\
\text { integration (TBI) }\end{array}$ & $\begin{array}{l}\text { Datasets for various multi-omics data types } \\
\text { are first converted into intermediate forms, } \\
\text { which are united into a large input matrix; } \\
\text { then a predictive model is generated by } \\
\text { using the large input matrix. }\end{array}$ & $\begin{array}{l}\text { Unique variables such as patient identifiers } \\
\text { can be used to link multi-omics data types } \\
\text { and integrate a variety of continuous or } \\
\text { categorical data values. }\end{array}$ & $\begin{array}{l}\text { It may be challenging to } \\
\text { transform into intermediate } \\
\text { forms. }\end{array}$ \\
\hline
\end{tabular}




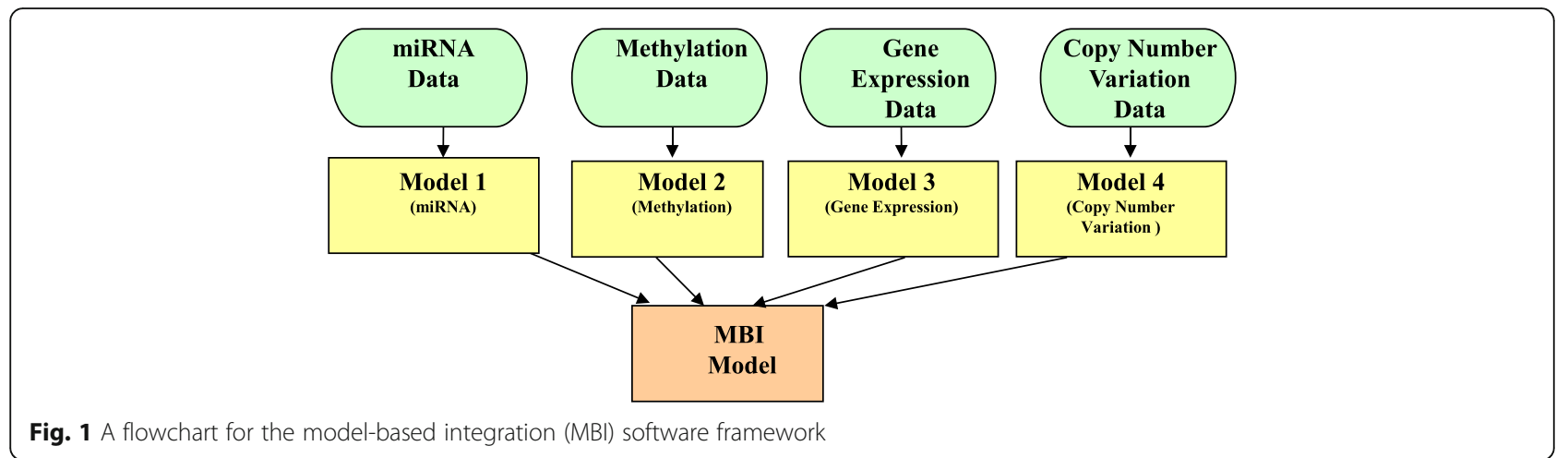

is a null network. Then, slight arbitrary changes are made to the network by flipping, adding, or deleting individual edges. Ultimately, accepting those changes will lead to an overall improvement by fitting the network to the data. In order to avoid over-fitting owing to the addition of new parameters, the Bayesian information criterion score [49] can be employed to assess whether a change improves the network model.

\section{Ensemble classifier framework}

In order to reduce the variance caused by the distinctiveness of a single genomic factor, Shen and Chou employed ensemble classifier models to integrate multiple classifiers, where each of those classifiers was based on individual genomic factor [50]. Thus, ensemble classifier models were able to obtain a more concrete concept in classification than a single classifier. The final output of the ensemble classifier model was the weighted fusion of the outputs generated by the individual basic classifiers. The weighted factor was assigned with the value of the success rate obtained by the individual basic classifier. Here, Shen and Chou adopted the optimized evidence-theoretic K-nearest-neighbors algorithm for the basic classifier [50].

\section{Concatenation-based integration approach}

Second, we investigated the CBI approach, which combines multiple data matrices for each dataset into one large input matrix before constructing a model (Fig. 2).

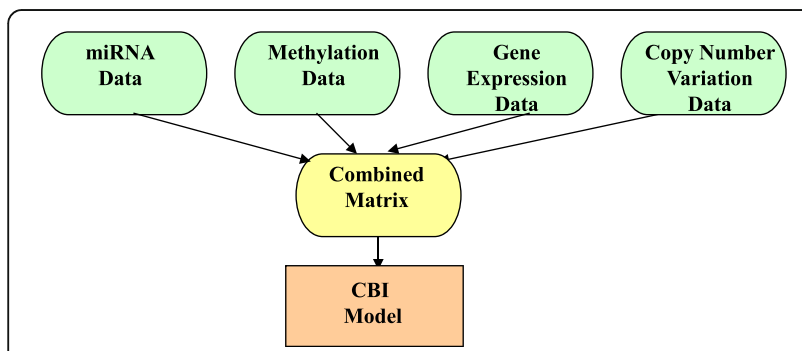

Fig. 2 A flowchart for the concatenation-based integration (CBI) software framework
One advantage of the CBI approach is that, after we determine how to combine all of the variables into one matrix, it is relatively simple to employ a variety of machine learning methods for analyzing continuous or categorical data [42].

In the literature, the CBI approach encompasses the following computational frameworks for constructing a model: Bayesian networks [51], multivariate Cox LASSO models [52], grammatical evolution neural networks [53], iCluster [54], Bayesian correlated clustering [55], and Bayesian consensus clustering [56]. In addition, We can consider some of the best-known machine learning algorithms including naive Bayes [19], C4.5 decision tree [20], ANNs [21-23], SVM [24], k-Means [25], kNN [26], and regression $[27,28]$. Depending on the number of variables in the data matrix, we can also employ data reduction and feature selection methods as described below.

In order to assess response to cancer therapeutics such as gemcitabine, Fridley et al. employed a Bayesian integrative model, which combines the ideas of Bayesian pathway analysis with Bayesian variable selection using stochastic search variable selection [51]. They employed two various high-throughput multi-omics datasets, such as mRNA expression and SNPs data, which were integrated into one large input matrix [51]. Fridley et al. reported that the Bayesian integrative model had greater sensitivity to detect genomic effects in the drug gemcitabine, as compared to the traditional single data type analysis [51].

Furthermore, instead of a single data type, Shen et al. implemented the iCluster framework to carry out cancer subtype discovery in glioblastoma using three multi-omics data types such as copy number data, mRNA expression data, and methylation data [54]. The iCluster framework is a CBI method that can simultaneously accomplish both data integration and dimension reduction to combine multi-omics data into one large input matrix [54]. Shen et al. revealed three distinct integrated tumor subtypes by using iCluster and multiomics data [54]. 


\section{Transformation-based integration approach}

Third, we assessed the TBI approach, which transforms each dataset into an intermediate form, such as a graph or a kernel matrix, and then merges multiple graphs or kernels into one before constructing a model (Fig. 3). One advantage of the TBI approach is that this approach can be employed to integrate a variety of continuous or categorical data values if the data contain unique variables such as patient identifiers for linking multi-omics data types [42].

In the literature, the TBI approach encompasses the following statistical frameworks for constructing a model: a kernel-based integration method [57] and a graph-based semi-supervised learning method [58]. The TBI approach investigates whether there is a relevant intermediate representation, such as a kernel or graph, for each multi-omics data type.

In order to find metabolic consequences underlying body weight change, Wahl et al. implemented a weighted correlation network approach [59], which was inferred using the Gaussian graphical model [60]. Instead of a single data type, they leveraged two different highthroughput multi-omics datasets, such as serum metabolomics and whole blood gene expression [59]. Wahl et al. first clustered multi-omics data into intermediate forms, namely modules of closely connected molecules, and then constructed a partial correlation network from the modules. Their analysis revealed that four metabolite and two gene expression modules were significantly associated with body weight change, indicating an association of long-term weight change with serum metabolite concentrations [59].

\section{Data reduction and feature selection approach}

Accounting for models is not a trivial task because even a relatively small set of factors results in the large number of possible models [61]. For example, if we study 10 factors, then these 10 factors yield $2^{10}$ possible models. The purpose of data reduction and feature selection approaches is to find a subset of factors that maximizes the performance of the prediction model, depending on how these methods incorporate the feature selection search with the classification algorithms. There are two data reduction and feature selection approaches including extrinsic approaches (which use information external to the data set itself) and intrinsic approaches (which use the data set and some analytical technique for filtering). The extrinsic approaches, such as Biofilter [62], employ prior knowledge that is accessible in the public domain. Additionally, the intrinsic approaches encompass factor analysis [63], ReliefF [64], chi-square statistics, principal component analysis [65], and genetic algorithms [66].

Furthermore, a hybrid approach, which combines the information-gain method and the chi-squared method, is designed to reduce bias introduced by each of the methods [67]. Each feature is measured and ranked according to its merit in both methods. The measurement of the merit for the two methods is defined as follows. The information-gain method measures the decrease in the entropy of a given feature provided by another feature, and the chi-squared method is based on Pearson chi-squared statistic to measure divergence from the expected distribution. Next, all features are sorted by their average rank across these two methods. After the features are ranked, the classifiers are utilized to add one feature at a time based on its individual ranking and then select the desired number of the top ranked features that provides the best predictive performance, respectively.

Moreover, in a wrapper-based feature selection approach, the feature selection algorithm acts as a wrapper around the classification algorithm. The wrapper-based feature selection approach conducts best-first search for a good subset using the classification algorithm itself as

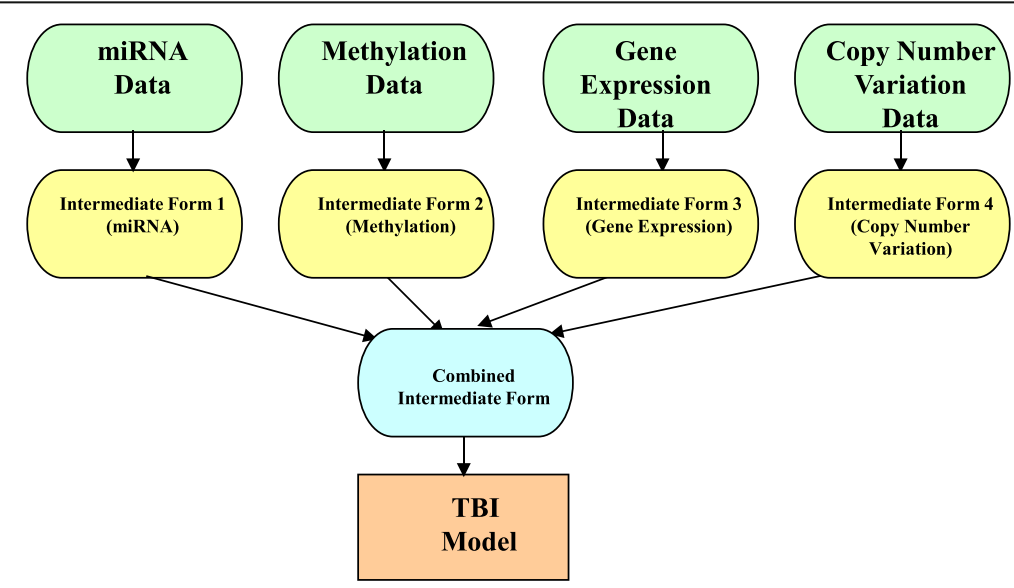

Fig. 3 A flowchart for the transformation-based integration (TBI) software framework 
part of the function for evaluating feature subsets $[68,69]$. Best first search starts with empty set of features and searches forward to select possible subsets of features by greedy hill-climbing augmented with a backtracking technique [18].

\section{Future perspective}

The MLSG modeling is essential to root out the false positive candidate genes discovered at the current association analyses by using meta-analysis, epistasis analysis, and pathway models [13]. Using multi-omics data not only could take care of missing information from any single data source, but also could help bridge the gap between phenotypes and more comprehensive biological regulation models [70]. In future research, models in MLSG will be established to predict the probability of drug efficacy to guide clinicians in choosing medications. In order to establish models for predicting drug efficacy, techniques in MLSG may provide a plausible way to predict drug efficacy in therapy. Finally, data analysis and integration in MLSG may play a key role in weighing gene-gene and gene-environment interactions.

\section{Conclusions}

In this study, we reviewed several recent findings and relevant studies in terms of the MLSG software frameworks. The work also underscores the importance of techniques in MLSG to track down a greater diversity of populations in the clinical settings of diseases and their treatments. In fact, facilitating the MLSG tools based on multi-omics data plays a pivotal role, economically and clinically, in predicting the possible outcomes of diseases and treatments. Future research using the MLSG approaches is needed in order to weigh the interplay among clinical factors and multi-omics data.

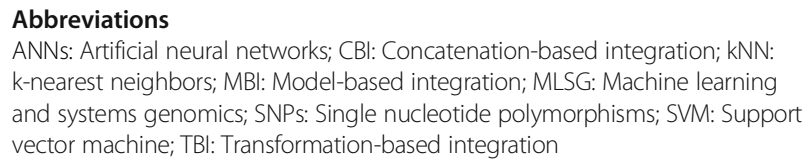

ANNs: Artificial neural networks; CBI: Concatenation-based integration; kNN: k-nearest neighbors; MBI: Model-based integration; MLSG: Machine learning and systems genomics; SNPs: Single nucleotide polymorphisms; SVM: Support vector machine; TBI: Transformation-based integration

\section{Acknowledgements}

Not applicable.

\section{Funding}

This work was supported by the Ministry of Economic Affairs in Taiwan (SBIR Grant S099000280249-154), Taiwan Ministry of Health and Welfare Clinical Trial and Research Center of Excellence (MOHW105-TDU-B-212-133019), and China Medical University Hospital, Taiwan (DMR-101-091 and DMR-102-069). The funding supports had no role in study design, data collection and analysis, decision to publish, or preparation of the manuscript.

\section{Availability of data and materials}

Not applicable.

\section{Authors' contributions}

The present manuscript was drafted by EL and revised by EL and HYL. Both authors read and approved the final manuscript.

\section{Competing interests}

Both authors declare that they have no competing interests.

\section{Consent for publication}

Not applicable.

Ethics approval and consent to participate

Not applicable.

\section{Author details}

${ }^{1}$ Graduate Institute of Biomedical Sciences, China Medical University, Taichung, Taiwan. ${ }^{2}$ Vita Genomics, Inc, Taipei, Taiwan. ${ }^{3}$ TickleFish Systems Corporation, Seattle, WA, USA. ${ }^{4}$ Department of Psychiatry, China Medical University Hospital, Taichung, Taiwan.

Received: 4 November 2016 Accepted: 3 January 2017 Published online: 20 January 2017

\section{References}

1. Katsanis SH, Javitt J, Hudson K. A case study of personalized medicine. Science. 2008;v320(4):53-4.

2. Snyderman R. Personalized health care: from theory to practice. Biotechnol J. 2012; $77: 973-9$

3. Lin E. Novel drug therapies and diagnostics for personalized medicine and nanomedicine in genome science, nanoscience, and molecular engineering. Pharma Regul Aff. 2012;1:e116.

4. Lin $E$, Lin CG, Wang JY, Wu LS. Gene-gene interactions among genetic variants from seven candidate genes with pediatric asthma in a Taiwanese population. Curr Topics Genet. 2009;3:83-8.

5. Lin E, Hong CJ, Hwang JP, Liou YJ, Yang CH, Cheng D, et al. Gene-gene interactions of the brain-derived neurotrophic-factor and neurotrophic tyrosine kinase receptor 2 genes in geriatric depression. Rejuvenation Res. 2009;12(6):387-93.

6. Lin $\mathrm{E}, \mathrm{Ts}$ sai $\mathrm{SJ}$. Gene-gene interactions in a context of individual variability in antipsychotic drug pharmacogenomics. Curr Pharmacogenomics Person Med. 2011;:323-31.

7. Lin E, Pei D, Huang YJ, Hsieh CH, Wu LS. Gene-gene interactions among genetic variants from obesity candidate genes for nonobese and obese populations in type 2 diabetes. Genet Test Mol Biomarkers. 2009;13(4):485-93.

8. Wu LS, Hsieh CH, Pei D, Hung YJ, Kuo SW, Lin E. Association and interaction analyses of genetic variants in ADIPOQ, ENPP1, GHSR, PPARgamma and TCF7L2 genes for diabetic nephropathy in a Taiwanese population with type 2 diabetes. Nephrol Dial Transplant. 2009;24(11):3360-6.

9. Wang CH, Ke WS, Lin E. Evaluation of the ENPP1 and PLIN single nucleotide polymorphisms with type 2 diabetes in a Taiwanese population: evidence for replication and gene-gene interaction. J Investig Med. 2012;60(8):1169-73.

10. Lin E, Tsai SJ. Novel diagnostics R\&D for public health and personalized medicine in Taiwan: current state, challenges and opportunities. Curr Pharmacogenomics Person Med. 2012;10:239-46.

11. Lin E, Hwang Y, Tzeng CM. A case study of the utility of the HapMap database for pharmacogenomic haplotype analysis in the Taiwanese population. Mol Diagn Ther. 2006;10:367-70.

12. Lin $E$, Chen PS. Pharmacogenomics with antidepressants in the STAR*D study. Pharmacogenomics. 2008;9:935-46.

13. Lin $\mathrm{E}$, Lane HY. Genome-wide association studies in pharmacogenomics of antidepressants. Pharmacogenomics. 2015;16(5):555-66.

14. Lin E, Tsai SJ. Genome-wide microarray analysis of gene expression profiling in major depression and antidepressant therapy. Prog Neuropsychopharmacol Biol Psychiatry. 2016;64:334-40.

15. Kononenko I. Machine learning for medical diagnosis: history, state of the art and perspective. Artif Intell Med. 2001;23(1):89-109.

16. Lane HY, Tsai GE, Lin E. Assessing gene-gene interactions in pharmacogenomics. Mol Diagn Ther. 2012;16(1):15-27.

17. Landset S, Khoshgoftaar TM, Richter AN, Hasanin T. A survey of open source tools for machine learning with big data in the hadoop ecosystem. J Big Data. 2015;2:24.

18. Lin E, Tsai SJ. Machine learning and predictive algorithms for personalized medicine: from physiology to treatment. In: Turnbull A, editor. Personalized medicine. New York: Nova Science Publishers (in press).

19. Domingos P, Pazzani M. On the optimality of the simple Bayesian classifier under zero-one loss. Mach Learn. 1997;29:103-37. 
20. Quinlan JR. C4.5: programs for machine learning. San Francisco: Morgan Kaufmann Publishers; 1993.

21. Kung SY, Hwang JN. Neural networks for intelligent multimedia processing Proc IEEE. 1998:86:1244-72.

22. Bishop CM. Neural networks for pattern recognition. Oxford: Clarendon; 1995

23. Rumelhart DE, Hinton GE, William RJ. Learning internal representation by error propagation. In: Parallel distributed processing: explorations in the microstructure of cognition. Cambridge: MIT Press; 1996. p. 318-62.

24. Vapnik V. The nature of statistical learning theory. New York: Springer; 1995.

25. Lloyd SP. Least squares quantization in PCM. IEEE Trans Inform Theory (Special Issue on Quantization). 1982;IT-28:129-37.

26. Altman NS. An introduction to kernel and nearest-neighbor nonparametric regression. Am Stat. 1992;46(3):175-85.

27. Friedman J, Hastie T, Tibshirani R. Regularization paths for generalized linear models via coordinate descent. J Stat Softw. 2010;33(1):1-22.

28. Zou H, Hastie T. Regularization and variable selection via the elastic net. J R Stat Soc Series B Stat Methodol. 2005:67(suppl):301-20.

29. Kessler RC, Warner CH, Ivany C, Petukhova MV, Rose S, Bromet EJ, et al. Predicting suicides after psychiatric hospitalization in US army soldiers. JAMA Psychiatry. 2015;72(1):49-57.

30. Huang LC, Hsu SY, Lin E. A comparison of classification methods for predicting chronic fatigue syndrome based on genetic data. J Transl Med. 2009;7:81.

31. Lin $E$, Chen PS, Yang YK, Lee $I H$, Yeh TL, Gean PW, et al. Modeling shortterm antidepressant responsiveness with artificial neural networks. Open Access Bioinform. 2010;2:55-60.

32. Lin E, Tsai SJ. Genetics and suicide. In: Courtet P, editor. Understanding suicide - from diagnosis to personalized treatment. Switzerland: Springer; 2016.

33. Lin E, Hsu SY. Gender differences and pharmacogenomics with antidepressants in depression. In: Hernandez P, Alonso S, editors. Women and Depression. New York: Nova; 2009

34. Kim W, Kim KS, Lee JE, Noh DY, Kim SW, Jung YS, et al. Development of novel breast cancer recurrence prediction model using support vector machine. J Breast Cancer. 2012;15(2):230-8.

35. Tseng CJ, Lu CJ, Chang CC, Chen GD. Application of machine learning to predict the recurrence-proneness for cervical cancer. Neural Comput \& Applic. 2014;24(6):1311-6.

36. Chen YC, Ke WC, Chiu HW. Risk classification of cancer survival using ANN with gene expression data from multiple laboratories. Comput Biol Med. 2014;48:1-7.

37. Chang SW, Abdul-Kareem S, Merican AF, Zain RB. Oral cancer prognosis based on clinicopathologic and genomic markers using a hybrid of feature selection and machine learning methods. BMC Bioinformatics. 2013;14:170.

38. Rosado P, Lequerica-Fernández P, Villallaín L, Peña I, Sanchez-Lasheras F, de Vicente JC. Survival model in oral squamous cell carcinoma based on clinicopathological parameters, molecular markers and support vector machines. Expert Syst Appl. 2013;40(12):4770-6.

39. Lin E, Hwang Y, Wang SC, Gu ZJ, Chen EY. An artificial neural network approach to the drug efficacy of interferon treatments. Pharmacogenomics. 2006;7:1017-24.

40. Ke WS, Hwang Y, Lin E. Pharmacogenomics of drug efficacy in the interferon treatment of chronic hepatitis C using classification algorithms. Adv App Bioinform Chem. 2010;3:39-44.

41. Lin E, Hwang Y. A support vector machine approach to assess drug efficacy of interferon-alpha and ribavirin combination therapy. Mol Diagn Ther. 2008:12:219-23.

42. Ritchie MD, Holzinger ER, Li R, Pendergrass SA, Kim D. Methods of integrating data to uncover genotype-phenotype interactions. Nat Rev Genet. 2015; 16(2):85-97

43. Draghici S, Potter RB. Predicting HIV drug resistance with neural networks. Bioinformatics. 2003;19:98-107.

44. Akavia UD, Litvin O, Kim J, Sanchez-Garcia F, Kotliar D, Causton HC, et al. An integrated approach to uncover drivers of cancer. Cell. 2010;143:1005-17.

45. Zhu J, Sova P, Xu Q, Dombek KM, Xu EY, Vu H, et al. Stitching together multiple data dimensions reveals interacting metabolomic and transcriptomic networks that modulate cell regulation. PLoS Biol. 2012;10:e1001301.

46. Kim D, Li R, Dudek SM, Ritchie MD. ATHENA: identifying interactions between different levels of genomic data associated with cancer clinical outcomes using grammatical evolution neural network. BioData Min. 2013;6:23.

47. Pearl J. Probabilistic reasoning in intelligent systems: networks of plausible inference. San Mateo: Morgan Kaufmann Publishers; 1988.
48. Madigan D, York J. Bayesian graphical models for discrete data. Int Stat Rev. 1995:63:215-32.

49. Schwarz G. Estimating the dimension of a model. Ann Stat. 1978;6:461-4.

50. Shen HB, Chou KC. Ensemble classifier for protein fold pattern recognition. Bioinformatics. 2006:22(14):1717-22.

51. Fridley $B L$, Lund $S$, Jenkins $G D$, Wang LA. Bayesian integrative genomic model for pathway analysis of complex traits. Genet Epidemiol. 2012;36:352-9.

52. Mankoo PK, Shen R, Schultz N, Levine DA, Sander C. Time to recurrence and survival in serous ovarian tumors predicted from integrated genomic profiles. PLOS ONE. 2011;6:e24709

53. Holzinger ER, Dudek SM, Frase AT, Pendergrass SA, Ritchie MD. ATHENA: the analysis tool for heritable and environmental network associations. Bioinformatics. 2014;30:698-705.

54. Shen R, Mo Q, Schultz N, Seshan VE, Olshen AB, Huse J, et al. Integrative subtype discovery in glioblastoma using iCluster. PLoS ONE. 2012;7:e35236.

55. Kirk P, Griffin JE, Savage RS, Ghahramani Z, Wild DL. Bayesian correlated clustering to integrate multiple datasets. Bioinformatics. 2012;28:3290-7.

56. Lock EF, Dunson DB. Bayesian consensus clustering. Bioinformatics. 2013;29: 2610-6.

57. Lanckriet GRG, De Bie T, Cristianini N, Jordan MI, Noble WS. A statistical framework for genomic data fusion. Bioinformatics. 2004;20:2626-35.

58. Shin $\mathrm{H}$, Lisewski AM, Lichtarge $\mathrm{O}$. Graph sharpening plus graph integration: a synergy that improves protein functional classification. Bioinformatics. 2007;23:3217-24.

59. Wahl S, Vogt S, Stückler F, Krumsiek J, Bartel J, Kacprowski T, et al. Multiomic signature of body weight change: results from a population-based cohort study. BMC Med. 2015;13:48.

60. Krumsiek J, Suhre K, Illig T, Adamski J, Theis FJ. Gaussian graphical modeling reconstructs pathway reactions from high-throughput metabolomics data. BMC Syst Biol. 2011;5:21.

61. Lin E, Huang LC. Identification of significant genes in genomics using Bayesian variable selection methods. Adv Appl Bioinform Chem. 2008;1:13-8.

62. Bush WS, Dudek SM, Ritchie MD. Biofilter: a knowledge-integration system for the multi-locus analysis of genome-wide association studies. Pac Symp Biocomput. 2009:368-379.

63. Hastie T, Tibshirani R, Friedman J. The elements of statistical learning: data mining, inference, and prediction. New York: Springer; 2001.

64. Greene CS, Penrod NM, Kiralis J, Moore JH. Spatially uniform ReliefF (SURF) for computationally efficient filtering of gene-gene interactions. BioData Min. 2009;2:5

65. Zou H, Hastie T, Tibshirani R. Sparse principal component analysis. J Comput Graph Stat. 2006;15:265-86.

66. Holland JH. Genetic algorithms. Sci Am. 1992;267:66-72.

67. Saeys $Y$, Inza I, Larrañaga P. A review of feature selection techniques in bioinformatics. Bioinformatics. 2007;23:2507-17.

68. Kohavi R, John GH. Wrappers for feature subset selection. Artif Intell. 1997; 97:273-324

69. Lin $\mathrm{E}$, Hwang $Y$, Liang $\mathrm{KH}$, Chen EY. Pattern-recognition techniques with haplotype analysis in pharmacogenomics. Pharmacogenomics. 2007;8(1):75-83.

70. Leung MKK, Delong A, Alipanahi B, Frey BJ. Machine learning in genomic medicine: a review of computational problems and data sets. Proc IEEE. 2016;104(1):176-97.

\section{Submit your next manuscript to BioMed Central and we will help you at every step:}

- We accept pre-submission inquiries

- Our selector tool helps you to find the most relevant journal

- We provide round the clock customer support

- Convenient online submission

- Thorough peer review

- Inclusion in PubMed and all major indexing services

- Maximum visibility for your research

Submit your manuscript at www.biomedcentral.com/submit 